\title{
Effect of Qihuang Decoction Combined with Enteral Nutrition on Postoperative Gastric Cancer of Nutrition and Immune Function
}

\author{
Qing-sheng Yu, ${ }^{1,2}$ Zhou Zheng $\mathbb{D}^{1,2}$ Hui Peng, ${ }^{1,2}$ Yi Shen, ${ }^{1,2}$ Ju-da Liu, ${ }^{1,2}$ and Fu-hai Zhou ${ }^{1,2}$ \\ ${ }^{1}$ Department of General Surgery, The First Affiliated Hospital of Anhui University of Chinese Medicine, Hefei, China \\ ${ }^{2}$ Institute of Chinese Medicine Surgery, Anhui University of Traditional Chinese Medicine, Hefei, China \\ Correspondence should be addressed to Zhou Zheng; 563382149@qq.com
}

Received 22 November 2019; Accepted 3 February 2020; Published 2 March 2020

Academic Editor: Gioacchino Calapai

Copyright $($ C 2020 Qing-sheng Yu et al. This is an open access article distributed under the Creative Commons Attribution License, which permits unrestricted use, distribution, and reproduction in any medium, provided the original work is properly cited.

Objective. Early nutritional support in patients with gastric cancer can improve their nutritional status, but the impact on immune function has not been confirmed. This study aimed to analyze the effects of Qihuang decoction combined with enteral nutrition on nutrition and the immune function of postoperative gastric cancer. Methods. 120 patients with postoperative gastric cancer in the study group and 117 in the control group were selected as the study subjects from our hospital at random. Indications of nutrition and immune and the rates of complications were compared the day before surgery and 1,3,7, and 14 days after surgery. Results. Indications of nutrition except hemoglobin (HB) in the study group were significantly higher than those before operation and the albumin (ALB) and prealbumin (TP) were significantly increased 7 and 14 days after surgery $(P<0.001$ and $P<0.001$ versus $P<0.001$ and $P<0.001)$ and the protein (PA) 3, 7, and 14 days after surgery $(P=0.011, P=0.002$, and $P=0.022)$ in the study group compared to those in the control group. Cellular and humoral immunity indications in the study group are significantly higher than those before operation compared to those in the control group, and the $\mathrm{CD} 3^{+}, \mathrm{CD} 4^{+}$, and $\mathrm{CD} 4^{+} / \mathrm{CD} 8^{+}$were significantly increased 7 and 14 days after surgery $(P=0.027$ and $P<0.001$ versus $P=0.008$ and $P<0.001$ versus $P=0.010$ and $P<0.001)$ and $\operatorname{IgA}, \operatorname{IgG}$, and $\operatorname{IgM} 3,7$, and 14 days after surgery in the study group $(P<0.001, P<0.001$, and $P<0.001$ versus $P<0.001, P<0.002$, and $P<0.001$ versus $P<0.001, P<0.001$, and $P<0.001)$. The complications such as abdominal, lung, wound, and urinary infection were also significantly decreased $\left(P \chi^{2}=0.017 ; P^{\chi^{2}}=0.036 ; P^{\chi^{2}}=0.041 ; P^{\chi^{2}}=0.004\right)$. Conclusions. Qihuang decoction combined with enteral nutrition can promote the absorption of enteral nutrition with improving the immune and reducing complications of infection.

\section{Introduction}

Gastric cancer is the third leading cause of cancer death in the world. Among the world's geographical regions, the highest incidence and mortality of gastric cancer are in Northeast Asian countries, including China, Japan, and South Korea, accounting for more than half of the world's total $[1,2]$. Owing to the characteristics of vigorous proliferation ability and autonomy of malignant tumor and the stress of preoperative fasting, surgery, and anesthesia, patients with gastric cancer have had cachexia such as marasmus, anemia, and other diseases by increasing the catabolism of the body and causing the body to be in negative nitrogen balance $[3,4]$. Malnutrition not only is not conducive to wound healing but also increases the incidence of complications and mortality in patients. Moreover, inhibition of immunity leads to metastasis and recurrence of tumors, so early nutritional support after gastric cancer surgery is particularly significant [5-7].

At present, the main methods of postoperative nutrition are parenteral nutrition (PN) and enteral nutrition (EN). However, PN can provide glucose, amino acid, and other nutrients for organs and tissues for postoperative patients. Owing to long-term digestive tract disposition, intestinal microecology is prone to disorder or bacterial translocate through atrophy of intestinal mucosa and disruption of the intestinal barrier, which not only increase enterogenic infection rate but also trigger systemic inflammatory response $[8,9]$. In contrast, EN can promote the growth and repair of damaged intestinal 
mucosal cells, maintain the balance and growth of the inherent flora in the gastrointestinal mucosa, and stimulate the secretion and releases of various related hormones so that it helps the recovery of the gastrointestinal motility [10-12]. However, gastroreflux aspiration pneumonia and short-term gastrointestinal symptoms occur frequently, such as diarrhea which may result in loss of nutrients and imbalance of water, electrolyte, acid, and base $[13,14]$. Since the 1990s, some researchers have tried to promote the recovery of the intestinal mucosa by adding special nutrients such as arginine, glutamine, $\omega$-fatty acids, nucleosides, and nucleotides to the standard enteric nutrient solution. We notice that the immunity of patients was improved to some extent, but the nutritional status of patients did not have an obvious advantage [15].

Our previous animal experiments showed that Qihuang decoction not only promoted the recovery of intestinal immune barrier in rats after gastrectomy but also improved the mechanical barrier of intestinal mucosa [16]. This study is to discuss the nutritional status, immune function, gastrointestinal function recovery, and complications of postoperative patients with gastric cancer in the early stage after gastric cancer in our hospital through intranasal feeding of Qihuang decoction combined with enteral nutrition emulsion.

\section{Patients and Methods}

2.1. Ethics Statement. The study was approved by the Ethics Committee of the First Affiliated Hospital of Anhui University of Traditional Chinese Medicine and complied with the Helsinki Declaration. All participants gave written informed consent before collecting data.

2.2. Patient Population. A total of 237 patients were ultimately selected from the first affiliated hospital of Anhui University of Traditional Chinese Medicine on January 1, 2015, and December 31, 2018, for gastric cancer surgery, including 120 patients in the study group and 117 patients in the control group. Diagnostic criteria were in line with the relevant standards for the diagnosis of gastric cancer diagnostic criteria issued in Japan in 2010 [17]. Inclusion criteria were as follows: patients with gastric cancer diagnosed by gastroscopy and pathology were selected for surgical treatment and patients who had not used chemotherapy for half a year. Exclusion criteria were as follows: patients who had have gastrointestinal dysfunction, abnormal liver function, intestinal absorption, metabolic disorders, immune dysfunction, or digestive system diseases; patients with severe malnutrition $\left(\mathrm{BMI}<18 \mathrm{~kg} / \mathrm{m}^{2}\right)$; pregnant and lactating women; patients with severe accompanying diseases such as chronic cardiopulmonary insufficiency and chronic renal failure; and patients who had have a history of cerebral infarction less than 6 months, and radical surgery could not be performed owing to patients with distant metastases found during surgery.
2.3. Allocation to Groups. The 244 patients who were initially recruited were randomly divided into the study group and control group according to $1: 1$, and random numbers (range 0 to 1 ) were generated for 244 using SPSS21.0 software. Then, the rank was compiled, taking 1 to 122 as the study group and 123 to 244 as the control group, the 001-244 marked strips were placed in an opaque envelope, and the patients randomly selected the strips. Then, we grouped the extracted strips digitally. Sealed envelopes are supervised by a specially assigned person, and patients and medical staff are completely unaware of the data and research (Figure 1).

\subsection{Intervention}

2.4.1. Preoperative Preparation. Both groups of patients performed the same preparation before surgery. Fasting water and diet 24 hours before surgery and oral catharsis medication to diarrhea 8 hours before surgery were performed by all patients for preoperative bowel preparation. 30 minutes before surgery, the second-generation cephalosporin was used to prevent postoperative abdominal infection, and a jejunal nutrient tube (trade name: Fuerkai Nasogastric tube, standard number: YZB/Su0943-2014; the manufacturer: Nutricia Pharmaceuticals Wuxi Co., Ltd.) was inserted into the side hole of the lowermost part of the stomach tube. The surface of the two tubes is coated with paraffin oil. When the tube is intubated, the patient took a deep breath and swallows normally until the two tubes are inserted into the stomach cavity through the patient's nostrils (the depth is 50 to $60 \mathrm{~cm}$ ) and the syringe is pumped with gastric juice out. The stomach tube is fixed with a tether and the jejunal nutrition tube is fixed with a tape.

\subsubsection{Postoperative Treatment}

(1) Control Group. Enteral nutrition emulsion was offered (TPF $500 \mathrm{ml}$ approval number: National Pharmaceutical Standard H20040188, Ruixian, $28 \mathrm{~g}$ of protein, $29 \mathrm{~g}$ of fat, $94 \mathrm{~g}$ of carbohydrate, $10 \mathrm{~g}$ of dietary fiber, various minerals and vitamins, and total energy supply of $750 \mathrm{kcal}$ ); $0.9 \%$ sodium chloride $100 \mathrm{ml}$ was given at $16 \mathrm{~h}$ after operation, and mixed suspension that contains $250 \mathrm{ml} \mathrm{TPF}$ and $500 \mathrm{ml}$ of $0.9 \%$ sodium chloride was given $24 \mathrm{~h}$ after operation; mixed suspension that contains $500 \mathrm{ml}$ TPF and $250 \mathrm{ml}$ of $0.9 \%$ sodium chloride was given on the $3 \mathrm{rd}$ to 4 th day after operation; $1000 \mathrm{ml} \mathrm{TPF}$ of total nutrient solution was given on the 5 th day after operation and $1500 \mathrm{ml}$ TPF of total nutrient solution was given from the 6th to 7th day; if energy supply is insufficient, intravenous infusion would be carried out according to the patient's post-dose reaction, and the measurement through the enteral nutrition tube is instilled intermittently from "less to more" (input for $4 \mathrm{~h}$ as well as intermittent for $30 \mathrm{~min}$; the enteral nutrition tube was rinsed with physiological saline before infusion for fear of obstruction). This procedure is performed until the transition to a liquid diet at 9:00 am and 3:00 pm, the speed is gradually increased 


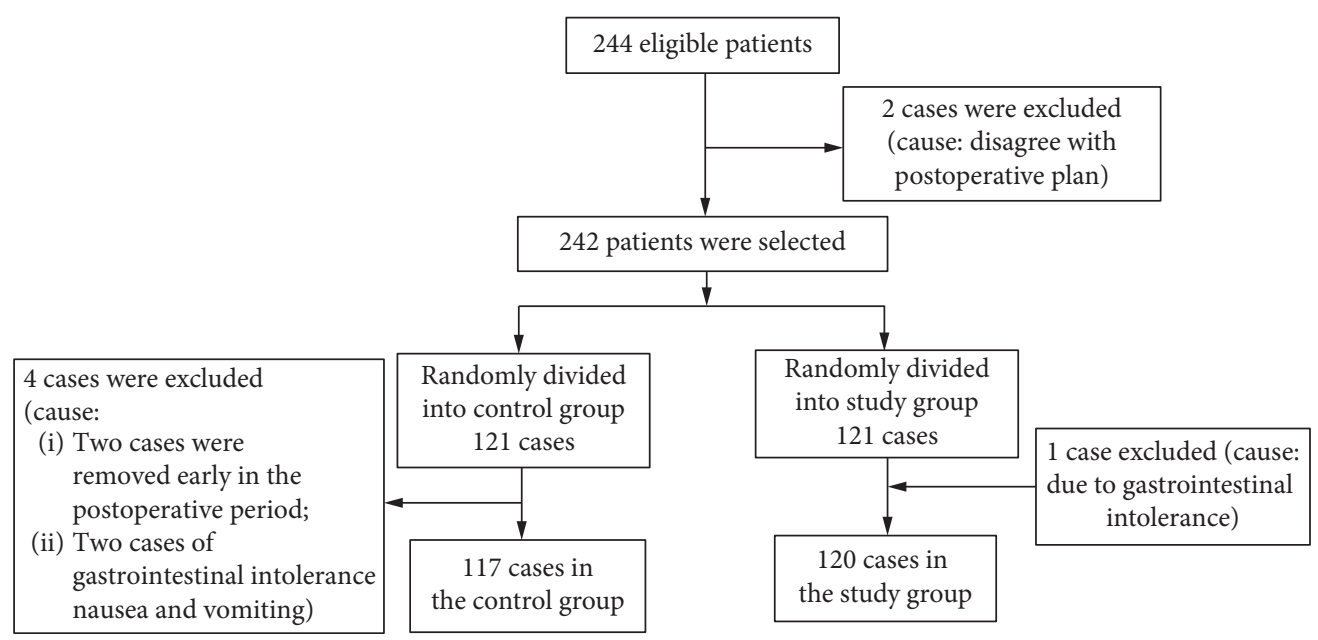

FIGURE 1: Diagram showing the flow of participants' enrollment.

from 10 or $20 \mathrm{drops} / \mathrm{min}$ to 40 or $60 \mathrm{drops} / \mathrm{min}$, and the temperature is controlled at 38 or $39^{\circ} \mathrm{C}$.

(2) Study Group. Qihuang decoction, which contains Astragalus membranaceus, Rheum officinale, rhizome of largehead atractylodes, Codonopsis pilosula, Fructus aurantii immaturus, Magnolia officinalis, Salvia miltiorrhiza, and Radix Scutellariae was offered to instill. They were mixed according to the mass ratio $20: 10: 20: 20: 10: 10: 15: 12$ and the total weight of mixed medicine was $234 \mathrm{~g} .500 \mathrm{ml} \mathrm{H} \mathrm{H}_{2} \mathrm{O}$ was added and boiled for $30 \mathrm{~min}$ according to [16]. The Crude Drug Decoction was filtrated and concentrated to $1.0 \mathrm{~g} / \mathrm{ml}$, and it was preserved at $4^{\circ} \mathrm{C}$ and rewarmed before administration. $150 \mathrm{ml}$ was infused every time, the temperature was 38 39, the speed was controlled at $30 \sim 40 \mathrm{ml} / \mathrm{min}$, and the total course of treatment was 7 days.

2.4.3. Observation Indicators and Their Detection. $3 \mathrm{ml}$ venous blood was taken from the median vein of the elbow at 6:00 in the morning before surgery and 3, 7, and 14 days after surgery. HB was detected by automatic blood cell analyzer (xn-9000, Sysmex) using Kurt method; ALB, TP, and PA were detected by special protein analyzer $(\mathrm{BN}$, Siemens) through BCG, biuret, and immunoturbidimetry; IgA, IgM, IgM, and $\mathrm{CD}^{+}, \mathrm{CD}^{+}$, and $\mathrm{CD} 4^{+} / \mathrm{CD}^{+}$were detected by automatic chemiluminescence assay (AutoLumo Awoo Plus, Zhengzhou Antu Biological Limited Company) using immunoturbidimetry and flow cytometry. Statistics of complications during hospitalization after gastric resection included anastomotic leakage, abdominal hemorrhage, abdominal infection, pulmonary infection, incision infection, urinary infections, gastroparesis syndrome, and early mortality and gastrointestinal motor function recovery after the operation in gastric cancer such as time of bowel sound recovery, anal exhaust time, and defecation time.

Anastomotic leakage was diagnosed by various clinical manifestations such as fever, abdominal pain, and peritonitis. Gastric juice and bile intestinal contents can be seen in abdominal drainage and this confirms the diagnosis combined with digestive tract iodine angiography [18, 19]. Abdominal hemorrhage was defined when the progressive decrease of hemoglobin was more than $20 \mathrm{~g} / \mathrm{L}$ in line with abdominal CT or color Doppler ultrasound [20]. Pulmonary infection was diagnosed when the body temperature $>37.5^{\circ} \mathrm{C}$, white blood cell count $>10 \times 10^{10} / \mathrm{L}$, and percentage of neutrophils $>90 \%$ combined with chest X-ray or CT [21]. Abdominal, incision, and urinary infection was proved when postoperative bacterial culture is positive [22]. Gastroparesis syndrome was diagnosed by delayed gastric emptying ruling out no mechanical obstruction and gastric drainage daily more than $800 \mathrm{ml}$ that lasts 10 days [23]. Death diagnosis is as follows: coma, brain reflexes, and apnea experiments show positive combined with an electrocardiogram [24]. Recovery time of bowel sounds, anal exhaust, and defecation was as follows: the time of onset of symptoms is recorded by the nurse and was given to the clinician in a written form, which used the time of record minus the end of surgery.

2.5. Statistical Analyses. Data analysis was performed using statistical software SPSS21.0 (SPSS Inc., Chicago, IL, USA). The measurement data was expressed as $\bar{x} \pm s$. When the data satisfies the normal distribution, the repeated measures analysis of variance was used. Otherwise, the Man$\mathrm{n}$-Whitney $U$ rank-sum test was used. The count data is expressed as a number of cases or as a percentage, and the comparison is checked by the Chi-square test or Fisher's exact test. $P<0.05$ was considered to be statistically significant.

\section{Results}

3.1. Baseline Characteristics. The postoperative hospitalization of 237 patients with gastric cancer was analyzed. Among them, 120 patients were given postoperative basic data of Qihuang decoction combined with enteral nutrition and 117 patients with normal saline combined with enteral nutrition. Gender, mean age, tumor site, 
TABLE 1: General information of patients with gastric cancer in the two groups.

\begin{tabular}{|c|c|c|c|c|}
\hline Group & Study group $(n=120)$ & Control group $(n=117)$ & $t / \chi^{2}$ & $P$ \\
\hline \multicolumn{5}{|l|}{ Gender } \\
\hline Male/female & $81 / 39$ & $96 / 21$ & 3.087 & 0.079 \\
\hline Mean age (mean $\pm S D$, years $)$ & $65.33 \pm 11.41$ & $66.75 \pm 9.00$ & -1.070 & 0.286 \\
\hline \multicolumn{5}{|l|}{ Tumor site } \\
\hline Cardiac region & 12 & 10 & & \\
\hline Fundus of stomach & 28 & 25 & 0.936 & 0.817 \\
\hline Gastric body & 38 & 34 & & \\
\hline Antrum of stomach & 42 & 48 & & \\
\hline \multicolumn{5}{|l|}{ Pathological stage(PTNM) } \\
\hline Stage I & 32 & 28 & & \\
\hline Stage II & 42 & 39 & 0.717 & 0.869 \\
\hline Stage III & 36 & 41 & & \\
\hline Stage IV & 10 & 9 & & \\
\hline \multicolumn{5}{|l|}{ Histological grading } \\
\hline Well & 55 & 44 & & \\
\hline Moderately & 42 & 48 & 1.668 & 0.434 \\
\hline Poorly & 23 & 25 & & \\
\hline \multicolumn{5}{|l|}{ Surgical method } \\
\hline Distal gastrectomy & 38 & 33 & 0.338 & 0.561 \\
\hline Total gastrectomy & 82 & 84 & & \\
\hline Time of operation(min) & $214.19 \pm 8.24$ & $213.29 \pm 6.09$ & 0.959 & 0.338 \\
\hline Intraoperative blood loss $(\mathrm{ml})$ & $220.06 \pm 8.75$ & $221.09 \pm 7.76$ & -0.957 & 0.340 \\
\hline
\end{tabular}

pathological stage, histological grading, surgical method, operative time, and intraoperative blood loss were recorded in Table 1.

3.2. Comparison of Nutritional Status in Both Groups. The ALB, TB, and PA in the two groups on Day 1 and Day 3 after operation were lower than those before the operation, and the decrease was most obvious after Day $1(P<0.05)$, but Days 7 and 14 after operation were significantly higher than those after Day $1(P<0.05)$. In the study group, ALB, TB, and PA 14 days after surgery were significantly higher than those before surgery $(P<0.05)$. There was no significant difference in $\mathrm{HB}, \mathrm{ALB}, \mathrm{TB}$, and PA between the two groups 1 day before the operation. On Days 7 and 14 after surgery, the levels of ALB and TB in the study group were significantly higher than those in the control group $(P<0.05)$ and on Days 3, 7, and 14 after surgery, the levels of PA were significantly higher $(P<0.05)$. However, no statistical significance was found in HB between the two groups on Days 1, 3,7 , and 14 after surgery $(P<0.05)$ as shown in Table 2.

3.3. Comparison of Immune Function in Both Groups. $\mathrm{CD}^{+}, \mathrm{CD}^{+}, \mathrm{CD}^{+} / \mathrm{CD}^{+}, \mathrm{IgA}, \mathrm{IgM}$, and $\operatorname{IgG}$ in the two groups were significantly lower on Day 1 after the operation than those before the operation $(P<0.05)$, but the levels of $\mathrm{CD}^{+}, \mathrm{CD}^{+}{ }^{+}, \mathrm{CD}^{+} / \mathrm{CD} 8, \operatorname{IgA}, \operatorname{IgM}$, and $\operatorname{IgG}$ were significantly higher on Days 3, 7, and 14 after the operation than those on Day 1 after the operation $(P<0.05)$. Moreover, $\mathrm{CD}^{+}, \mathrm{CD}^{+}$, $\mathrm{CD}^{+} / \mathrm{CD} 8, \mathrm{IgA}, \mathrm{IgM}$, and IgG in the study group exceeded the preoperative level on Day 14 after the operation $(P<0.05)$. Compared with the control group, IgA, IgM, and IgG increased significantly on Days 3,7 , and 14 after the surgery $(P<0.05)$, but $\mathrm{CD}^{+}, \mathrm{CD}^{+}$, and $\mathrm{CD} 4^{+} / \mathrm{CD} 8$ increased significantly on Days 7 and 14 after surgery only $(P<0.05)$ as shown in Table 3.

3.4. Gastrointestinal Recovery and Complications in Both Groups. The rates of pulmonary infection, abdominal infection, incision infection, and urinary infection in the study group were significantly lower than those in the control group and the differences were statistically significant $(P<0.05)$. Although the rates of anastomotic leakage, abdominal hemorrhage, gastroparesis syndrome, and early mortality in the study group were lower than those in the control group, the difference was not statistically significant $(P>0.05)$ as shown in Table 4 .

\section{Discussion}

Gastric cancer, the gastric epithelial malignant tumor, ranks fifth and second in death in the incidence of malignant tumors [25]. Its clinical manifestations are not specific. Early symptoms are only the upper abdomen being full of discomfort or dull pain, loss of appetite, and malignant vomiting and late symptoms include weight loss, fever, jaundice, and other cachexias, which are often ignored by patients. Therefore, active measures should be taken early in the clinical stage. Until now, surgery is still the main method of treatment for gastric cancer. However, partial gastrointestinal neurotomy and extensive resection of organs and tissues, as well as reconstruction of the digestive tract all together can lead to postoperative gastric motility and motor dysfunction by the disordering of gastrointestinal hormone secretion, so the patient's presentation is postoperative bloating, abdominal pain, and indigestion [26]. At the same time, surgical trauma, pathophysiological changes, and 


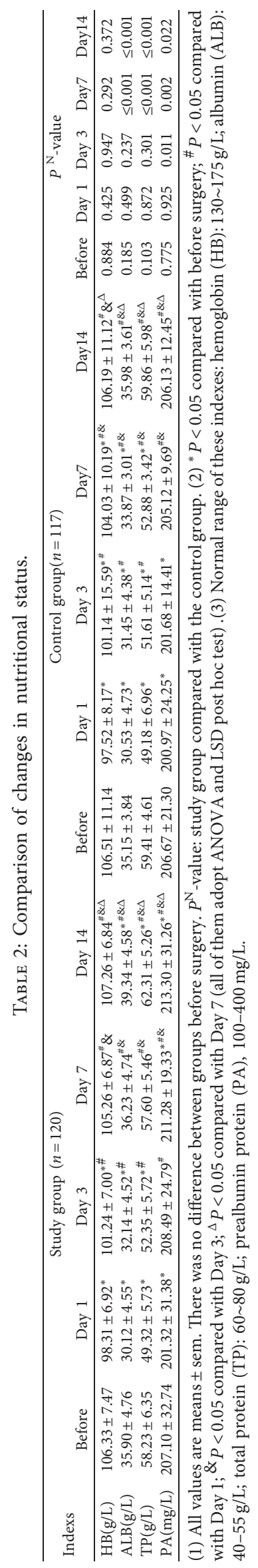


TABLE 3: Comparison of immune function.

\begin{tabular}{|c|c|c|c|}
\hline & Study group $(n=120)$ & Control group $(n=117)$ & $P^{N}$-value \\
\hline \multicolumn{4}{|c|}{$\mathrm{CD3}^{+}$(\%) normal range: $60 \% \sim 80 \%$} \\
\hline Before & $50.11 \pm 7.53$ & $49.42 \pm 5.37$ & 0.419 \\
\hline Day 1 & $43.13 \pm 7.64^{*}$ & $42.67 \pm 4.07^{*}$ & 0.567 \\
\hline Day 3 & $47.34 \pm 7.59^{* \#}$ & $46.06 \pm 3.45^{* \#}$ & 0.097 \\
\hline Day 7 & $48.12 \pm 7.32^{* \#}$ & $46.01 \pm 7.25^{* \#}$ & 0.027 \\
\hline Day 14 & $52.20 \pm 7.48^{* \# \& \Delta}$ & $48.78 \pm 7.06^{\# \& \Delta}$ & $\leq 0.001$ \\
\hline F-test & 4.069 & $P^{M}$-value & 0.008 \\
\hline \multicolumn{4}{|c|}{$\mathrm{CD}^{+}$(\%) normal range: $35 \% \sim 55 \%$} \\
\hline Before & $29.00 \pm 4.73$ & $29.49 \pm 4.22$ & 0.404 \\
\hline Day 1 & $23.33 \pm 3.64^{*}$ & $24.42 \pm 5.35^{*}$ & 0.412 \\
\hline Day 3 & $27.01 \pm 4.74^{* \#}$ & $26.43 \pm 5.24^{* \#}$ & 0.370 \\
\hline Day 7 & $31.89 \pm 4.09^{* \# \&}$ & $30.05 \pm 6.28^{\#}$ & 0.008 \\
\hline Day 14 & $34.00 \pm 4.66^{* \# \& \Delta}$ & $30.64 \pm 5.93^{* \#}$ & $\leq 0.001$ \\
\hline F-test & 9.990 & $P^{M}$-value & $\leq 0.001$ \\
\hline \multicolumn{4}{|c|}{$\mathrm{CD}^{+} / \mathrm{CD}^{+}$normal range: $1.4 \sim 2.0$} \\
\hline Before & $0.79 \pm 0.45$ & $0.88 \pm 0.27$ & 0.098 \\
\hline Day 1 & $0.70 \pm 0.40^{*}$ & $0.75 \pm 0.15^{*}$ & 0.205 \\
\hline Day 3 & $0.82 \pm 0.39^{\#}$ & $0.80 \pm 0.35^{* \#}$ & 0.731 \\
\hline Day 7 & $0.94 \pm 0.38^{* \# \&}$ & $0.84 \pm 0.19^{\#}$ & 0.010 \\
\hline Day 14 & $1.43 \pm 0.64^{* \# \& \Delta}$ & $1.01 \pm 0.27^{* \# \& \Delta}$ & $\leq 0.001$ \\
\hline F-test & 48.682 & $P^{M}$-value & $\leq 0.001$ \\
\hline \multicolumn{4}{|c|}{ IgA $(g / L)$ normal range:0.70 4.06 $\mathrm{g} / \mathrm{L}$} \\
\hline Before & $1.62 \pm 0.38$ & $1.59 \pm 0.27$ & 0.420 \\
\hline Day 1 & $1.32 \pm 0.29^{*}$ & $1.31 \pm 0.16^{*}$ & 0.592 \\
\hline Day 3 & $2.03 \pm 0.53^{* \#}$ & $1.63 \pm 0.36^{\#}$ & $\leq 0.001$ \\
\hline Day 7 & $2.45 \pm 0.42^{* \# \&}$ & $1.83 \pm 0.19^{* \# \&}$ & $\leq 0.001$ \\
\hline Day 14 & $3.44 \pm 0.42^{* \# \& \Delta}$ & $2.67 \pm 0.21^{* \# \& \Delta}$ & $\leq 0.001$ \\
\hline F-test & 64.849 & $P^{\bar{M}}$-value & $\leq 0.001$ \\
\hline \multicolumn{4}{|c|}{$\operatorname{IgM}(\mathrm{g} / \mathrm{L})$ normal range:0.34 2.14 $\mathrm{g} / \mathrm{L}$} \\
\hline Before & $1.72 \pm 0.36$ & $1.66 \pm 0.30$ & 0.165 \\
\hline Day 1 & $1.10 \pm 0.43^{*}$ & $1.02 \pm 0.25^{*}$ & 0.079 \\
\hline Day 3 & $1.92 \pm 0.39^{* \#}$ & $1.30 \pm 0.44^{* \#}$ & $\leq 0.001$ \\
\hline Day 7 & $2.30 \pm 0.36^{* \# \&}$ & $1.42 \pm 0.22^{* \# \&}$ & $\leq 0.001$ \\
\hline Day 14 & $2.42 \pm 0.41^{* \# \& \Delta}$ & $1.90 \pm 0.12^{* \# \& \Delta}$ & $\leq 0.001$ \\
\hline F-test & 71.784 & $P^{M}$-value & $\leq 0.001$ \\
\hline \multicolumn{4}{|c|}{ IgG(g/L) normal range:6.80 14.50 g/L } \\
\hline Before & $9.32 \pm 3.86$ & $9.64 \pm 2.23$ & 0.442 \\
\hline Day 1 & $6.30 \pm 3.53^{*}$ & $6.02 \pm 0.32^{*}$ & 0.394 \\
\hline Day 3 & $7.12 \pm 3.38^{* \#}$ & $6.32 \pm 1.51^{* \#}$ & 0.020 \\
\hline Day 7 & $8.35 \pm 1.44^{* \# \&}$ & $7.66 \pm 0.40^{* \# \&}$ & $\leq 0.001$ \\
\hline Day 14 & $10.44 \pm 3.31^{* \# \& \Delta \Delta}$ & $8.92 \pm 0.95^{* \# \& \Delta}$ & $\leq 0.001$ \\
\hline F-test & 10.257 & $P^{M}$-value & $\leq 0.001$ \\
\hline
\end{tabular}

(1) All values are means \pm sem. There was no difference between groups before surgery. (2) $P^{N}$-values for the difference between study group and control group with respect to the time point were calculated as treatment $\times$ time interaction. $P^{M}$-values for the difference between study group and control group with all the time points were calculated as treatment $\times$ time interaction. $(3)^{*} P<0.05$ compared with before surgery; ${ }^{\#} P<0.05$ compared with Day $1 ; \&_{P} P 0.05$ compared with Day $3 ;{ }^{\Delta} P<0.05$ compared with Day 7 (all of them adopt ANOVA and LSD post hoc test).

TABle 4: Postoperative complications $n(\%)$ in the study group and control group.

\begin{tabular}{|c|c|c|c|c|c|}
\hline Complication & Study group $(n=120)$ & Control group $(n=117)$ & Total $(n=237)$ & $\chi^{2}$ & $P$ \\
\hline Anastomotic leakage & $4(3.33)$ & $6(5.13)$ & $10(4.22)$ & - & $P^{f}=0.359$ \\
\hline Abdominal hemorrhage & $10(8.33)$ & $13(11.11)$ & $23(9.70)$ & 0.522 & $P^{X^{2}}=0.470$ \\
\hline Abdominal infection & $5(4.17)$ & $15(12.82)$ & $20(8.44)$ & 5.742 & $P^{X^{2}}=0.017$ \\
\hline Pulmonary infection & $12(10.00)$ & $23(19.66)$ & $35(14.77)$ & 4.39 & $P^{X^{2}}=0.036$ \\
\hline Incision infection & $7(5.83)$ & $16(13.68)$ & $23(9.70)$ & 4.157 & $P^{X^{2}}=0.041$ \\
\hline Urinary infection & $5(4.17)$ & $18(15.38)$ & $15(6.33)$ & 8.507 & $P^{X^{2}}=0.004$ \\
\hline Gastroparesis syndrome & $2(1.67)$ & $5(4.27)$ & $7(2.95)$ & - & $P^{f}=0.227$ \\
\hline Early mortality & $0(0)$ & $2(1.71)$ & $2(0.84)$ & - & $P^{f}=0.243$ \\
\hline
\end{tabular}

(1) $P^{X^{2}}$ : in Pearson Chi-square test; $P^{f}$ : in Fisher's exact test; $n$ : the number of examined patients. (2) $\chi^{2}$ : random variable Chi-square test value. 
postoperative stress will also aggravate the catabolism of the body, resulting in malnutrition and immunosuppression $[15,27]$. Malnutrition and low immune function lead to inflammatory reactions, which not only increase postoperative complications such as infection but also increase the recurrence of postoperative tumors and seriously affect the quality of life after surgery. Khorgami et al. found that immunosuppression and postoperative inflammatory response may lead to increased postoperative infection and tumor cell metastasis [28]. Esteban et al. have shown that the inflammation and immune status of gastric cancer patients are closely related to the occurrence of postoperative complications [29]. Therefore, the key to postoperative treatment is to select the appropriate nutritional support methods to improve the immune function of the body and correct malnutrition in time.

Early enteral nutrition has two advantages. Firstly, it can improve the recovery of intestinal peristaltic function by improving the height of intestinal villi, maintaining the mechanical barrier of the intestinal mucosa, protecting the growth of beneficial bacteria in the intestinal tract, and stimulating gastric acid secretion. Secondly, it is beneficial for liver protein synthesis and metabolism to promote early incision healing $[30,31]$. Currently, the most popular ways are standard enteral nutrition and immune enteral nutrition. Some scholars believe that standard enteral nutrition support can correct the nutrition-related complications of patients, but the immune and inflammatory response is not obvious [15]. ESPEN (European Society of Parenteral Enteral Nutrition) recommends that patients with upper gastrointestinal tumors use immune enteral nutrition (glutamine, arginine, omega-3 fatty acids, and nucleotides) to promote lymphocyte proliferation and differentiation to improve immune function, shorten hospital stay, and control postoperative infection. However, whether immune intestinal nutrition is superior to standard enteral nutrition in terms of immune indicators remains controversial [27]. The research on the effects of traditional Chinese medicine combined with enteral nutrition support on the nutritional status and immune function of postoperative patients with gastric cancer has not been deepened. Previous animal experiments in our group have shown that Chinese herbal medicine of Qihuang decoction can not only improve the nutritional status of rats after gastrectomy but also increase the number of $\mathrm{T}$ cells and $\mathrm{B}$ cells in epithelial lymphocytes and lamina propria lymphocytes [32]. Our study provides a bold attempt to study the effects of Chinese herbal medicine of Qihuang decoction combined with EN on the nutritional and immune status of patients with postoperative gastric cancer.

Visceral protein is the most important nutritional monitoring index, including albumin, prealbumin, and total protein. Malnutrition has existed in the patients because of tumor consumption and tumor body release of toxins [33]. This study showed that the preoperative $\mathrm{HB}$, $\mathrm{ALB}, \mathrm{TB}$, and PA were lower than normal. The surgical trauma, pathophysiological changes, and postoperative stress led to more catabolism in the body. The most significant decrease was also observed from our research on Day 1 after surgery $(P<0.05)$, which arrived at the same conclusion as they have. Prealbumin has a short half-life and good specificity, which can reflect the nutritional status and prognosis of patients [33]. The study showed that ALB and TB were significantly higher in the study group than those in the control group on Days 7 and 14 after surgery $(P<0.05)$, while $\mathrm{PA}$ in the study group was significantly higher than that in the control group on Days 3, 7, and 14 after surgery $(P<0.05)$. The analysis of nutritional status improvement reasons was as follows. On the one hand, gastrin, gastric acid, hormones, and enzymes can be more promoted in Qihuang decoction combined with enteral nutrition, which contributes to promoting the recovery of gastrointestinal motility and function and shortening the fasting time of patients. On the other hand, Qihuang decoction inhibits cell apoptosis and alleviates the injury caused by intestinal mucosal epithelium induced by ischemia reperfusion through upregulating Bcl-2 mRNA and downregulating the expressions of Bax, Caspase 3, and Caspase $9 \mathrm{mRNA}$, and this is more conducive to the absorption of intestinal nutrition [31, 34, 35]. Owing to the stress of surgery, intraoperative blood loss, and the inability to recover hematopoietic organs in a short time, the increase of $\mathrm{HB}$ is not obvious $(P>0.05)$.

Surgical stress induces neuroendocrine responses that promote the release of hormones such as catecholamine (norepinephrine and adrenaline), corticotrophin, and cortisol by activating the sympathetic nervous system and the hypothalamic-pituitary-adrenal HPA axis, which suppress the immune response [36]. Among them, $\mathrm{CD}^{+}$is a kind of T cells, which is also the basis of cellular immunity. $\mathrm{CD} 4^{+}$ belongs to a kind of helper T cells. The importance of $\mathrm{CD} 4^{+}$ cells in coordinating the immune response has increased significantly over the past decade. IgA, IgG, and IgM are mainly secreted by B lymphocytes to exert humoral immunity [37]. The body's immune surveillance is completed by T cells and B cells, reflecting the body's immune function and disease development. Yu et al. found that the postoperative cellular and humoral immunity of patients treated with Qihuang decoction increased significantly [38]. We found that the $\mathrm{CD}^{+}, \mathrm{CD}^{+}, \mathrm{CD}^{+} / \mathrm{CD} 8, \operatorname{IgA}, \mathrm{IgM}$, and $\mathrm{IgG}$ in the study group exceeded the preoperative level on Day 14 after the operation $(P<0.05)$. We also found that IgA, IgM, and IgG in the study group increased significantly on Days 3, 7, and 14 after operation compared with the control group $(P<0.05)$, but $\mathrm{CD}^{+}, \mathrm{CD}^{+}$, and $\mathrm{CD} 4^{+} / \mathrm{CD} 8$ increased significantly on Days 7 and 14 after surgery only $(P<0.05)$. At the same time, infectious incidences like pulmonary, abdominal, incision, and urinary system infection were also significantly reduced $(P<0.05)$. It further indicated that Qihuang decoction combined with EN reduced the incidence of complications significantly by promoting both cellular and humoral immunity, especially humoral immunity recovered earlier. Its possible mechanism is that Qihuang decoction controls inflammatory response and regulates immunity by inhibiting proinflammatory cytokines (IL-2 $\alpha$, IL-4, and IL-10) and upregulating anti- 
inflammatory cytokines (IL- $1 \alpha$, IL- 6 , and TNF- $\alpha$ ). On the other hand, it has adjusted to the whole body humoral and cellular immune function, which could be explained with the effect site (mucosa lamina propria and intraepithelial) and parts of the sensitization collection of the lymphoid tissue (Peyer's patches (PP)). They mainly regulate the level of lymphocyte homing in the aggregated lymphoid tissue (PP) and mesenteric lymph nodes, so that the lymphocyte level in the blood rises to play the role of $\mathrm{T}$ lymphocytes and B lymphocytes [32, 39].

Therefore, our study showed that Qihuang decoction combined with EN could make up for the deficiency of EN alone. Astragalus polysaccharide can induce apoptosis of gastric cancer MGC-803 cells by blocking the S phase cell cycle and interfering with the mitochondrial intrinsic apoptotic pathway from modern pharmacological studies [40]. Atractylodes polysaccharide can promote the lymphocyte into the $\mathrm{S}$ phase and $\mathrm{G} 2 / \mathrm{M}$ phase and increase the concentration of $\mathrm{CD}^{+}$and $\mathrm{CD}^{+}$in $\mathrm{T}$ lymphocytes and it is positively correlated with its concentration [41]. Rhubarb can enhance the innate immune homeostasis of the host mucosa by increasing the height of villi in the ileum, upregulating anti-inflammatory factor IL-10, reducing the proinflammatory factor IL- $1 \beta$ in the jejunum and ileum, and promoting the increase of claudin-1 mRNA and protein expression [42]. We in previous studies have confirmed that the fingerprint of Qihuang decoction has shared peak data information to ensure that there is no difference in the composition and efficacy in them where the main component of water-soluble saponin is baicalin. We have confirmed that baicalin enhanced the intestinal immune barrier mainly by promoting the proliferation and differentiation of intestinal mucosal lymphocytes and the synthesis and secretion of immunoglobulin by intestinal mucosal cells after gastrectomy in rat experiments. At the same time, the improvement of the mechanical barrier was also achieved by inhibiting the phosphorylation level of tight junction proteins [16].

\section{Conclusions}

Patients with gastric cancer after surgery are suffering from stress such as surgery and anesthesia, which can enhance the body's catabolism and lead to malnutrition and immunosuppression of patients that increase the risk of postoperative infection and other complications. Early treatment of gastric cancer after the treatment with Qihuang decoction combined with EN does not increase the incidence of complications such as anastomotic leakage and abdominal bleeding but reduces the incidence of infection. At the same time, it can accelerate the recovery of nutrition and immune function. We avoid abdominal distension and diarrhea caused by the infusion to adopt control of the infusion speed and temperature from less to more and slow to fast. Due to the limited sample size and selection of indicators this time, we will further refine the indicators and increase the sample size and further study the impact of Qihuang decoction on inflammatory indicators.

\section{Data Availability}

The data used to support the findings of the study are available from the corresponding author upon request.

\section{Conflicts of Interest}

The authors declare that they have no conflicts of interest.

\section{Authors' Contributions}

Zhou Zheng contributed to the study design. Hui Peng, Yi Shen, Ju-da Liu, and Fu-hai Zhou contributed to data collection and analysis. The manuscript was drafted by Qingsheng Yu. All authors have read and approved the final manuscript.

\section{Acknowledgments}

The authors would like to acknowledge the financial assistance of the National Natural Science Foundation of China (nos. 81573987, 30973855, and 81403406), Supporting Program for Excellent Young Talents in Colleges and Universities of Anhui Province (no. gxyq2019035), and 12th Five-Year National Key Clinical Specialist Construction Project (no. [2013] 239).

\section{References}

[1] International Agency for Research on Cancer, Stomach Cancer, GLOBOCAN, Lyon, France, 2012.

[2] K. Kimura, "Gastritis and gastric cancer Asia," Gastroenterology Clinics of North Ameria, vol. 29, no. 3, pp. 609-621, 2000.

[3] M. Holst, H. H. Rasmussen, and B. S. Laursen, "Can the patient perspective contribute to quality of nutritional care?" Scandinavian Journal of Caring Sciences, vol. 25, no. 1, pp. 176-184, 2011.

[4] S. C. H. M. Dos, O. M. Gomes, P. J. C. D. Vieira et al., "The ischemic preconditioning and postconditioning effect on the intestinal mucosa of rats undergoing mesenteric ischemia/ reperfusion procedure," Acta Cirurgica Brasileira, vol. 23, no. 1, pp. 22-28, 2008.

[5] A. Weimann, M. Braga, L. Harsanyi et al., "ESPEN guidelines on enteral nutrition: surgery including organ transplantation," Clinical Nutrition, vol. 25, no. 2, pp. 224-244, 2006.

[6] M. Braga, O. Ljungqvist, P. Soeters, K. Fearon, A. Weimann, and F. Bozzetti, "ESPEN guidelines on parenteral nutrition: surgery," Clinical Nutrition, vol. 28, no. 4, pp. 378-386, 2009.

[7] O. Ciacio, T. Voron, G. Pittau et al., "Interest of preoperative immunonutrition in liver resection for cancer: study protocol of the PROPILS trial, a multicenter randomized controlled phase IV trial," BMC Cancer, vol. 14, no. 1, p. 980, 2014.

[8] W. Karcz, S. Głuszek, and M. Kot, "Influence of nutritional treatment on the postoperative course in patients with gastric cancer," Advances in Medical Sciences, vol. 51, pp. 278-282, 2006.

[9] J. V. Peter, J. L. Moran, and J. Phillips-Hughes, "A meta analysis of treatment outcomes of early enteral versus early parenteral nutrition in hospitalized patients," Critical Care Medicine, vol. 33, no. 1, pp. 213-220, 2005.

[10] J. Peng, J. Cai, Z.-X. Niu, and L.-Q. Chen, "Early enteral nutrition compared with parenteral nutrition for esophageal 
cancer patients after esophagectomy: a meta-analysis," Diseases of the Esophagus, vol. 29, no. 4, pp. 333-341, 2016.

[11] R. Lee Koretz, "Early enteral nutrition in the ICU," Intensive Care Medicine, vol. 36, no. 6, pp. 1087-1088, 2010.

[12] M. Holst, H. H. Rasmussen, and O. Irtun, "Advances in clinical nutrition in GI surgery," Expert Review of Gastroenterology \& Hepatology, vol. 9, no. 4, pp. 467-473, 2015.

[13] M. Kozeniecki and R. Fritzshall, "Enteral nutrition for adults in the hospital setting," Nutrition in Clinical Practice, vol. 30, no. 5, pp. 634-651, 2015.

[14] K. Whelan and S. M. Schneider, "Mechanisms, prevention, and management of diarrhea in enteral nutrition," Current Opinion in Gastroenterology, vol. 27, no. 2, pp. 152-159, 2011.

[15] K. Li, Y. Xu, Y. Hu, Y. Liu, X. Chen, and Y. Zhou, "Effect of enteral immunonutrition on immune, inflammatory markers and nutritional status in gastric cancer patients undergoing gastrectomy: a randomized double-blinded controlled trial," Journal of Investigative Surgery, vol. 19, pp. 1-10, 2019.

[16] H. Peng, Y. Shen, Q. Zhang et al., "Qihuang decoction promotes the recovery of intestinal immune barrier dysfunction after gastrectomy in rats," American Journal of Translational Research, vol. 10, no. 3, pp. 827-836, 2018.

[17] The Precised Management of Surgical Treatment for Gastric Cancer, "Interpretation of the 5th edition of Japanese gastric cancer treatment guideline and the $15^{\text {th }}$ edition of Japanese classification for gastric cancer," Zhonghua Zhong Liu Za Zhi, vol. 41, no. 3, pp. 168-172, 2019.

[18] R. Hompes, S. Arnold, G. Wynn et al., "Incidence and risk factors for anastomoticfailure in 1594 patients treated by transanal total mesorectal excision:results from the international TaTME registry," Annals of Surgery, vol. 269, no. 4, pp. 700-711, 2019.

[19] J. R. Siewert, H. J. Stein, and H. Bartels, "[Anastomotic leaks in the upper gastrointestinal tract]," Der Chirurg, vol. 75, no. 11, pp. 1063-1070, 2004.

[20] J.-P. Tasu, G. Vesselle, G. Herpe et al., "Postoperative abdominal bleeding," Diagnostic and Interventional Imaging, vol. 96, no. 7-8, pp. 823-831, 2015.

[21] D.-W. Li, C.-Y. Du, B. Fan, P. Huang, S.-Q. Luo, and Q. He, "Impact of simultaneous splenectomy and orthotopic liver transplantation in patients with end-stage liver diseases and splenic hyperfunction," Hepatobiliary \& Pancreatic Diseases International, vol. 11, no. 5, pp. 489-493, 2012.

[22] J. S. Solomkin, J. E. Mazuski, J. S. Bradley et al., "Diagnosis and management of complicated intra-abdominal infection in adults and children: guidelines by the Surgical Infection Society and the Infectious Diseases Society of America," Surgical Infections, vol. 11, no. 1, pp. 79-109, 2010.

[23] B. Stein, K. K. Everhart, and B. E. Lacy, "Gastroparesis: a review of current diagnosis and treatment options," Journal of Clinical Gastroenterology, vol. 49, no. 7, pp. 550-558, 2015.

[24] E. F. M. Wijdicks, P. N. Varelas, G. S. Gronseth, and D. M. Greer, "Evidence-based guideline update: determining brain death in adults: report of the Quality Standards Subcommittee of the American Academy of Neurology," Neurology, vol. 74, no. 23, pp. 1911-1918, 2010.

[25] F. Bray, J. Ferlay, I. Soerjomataram, R. L. Siegel, L. A. Torre, and A. Jemal, "Global cancer statistics 2018: GLOBOCAN estimates of incidence and mortality worldwide for 36 cancers in 185 countries," CA: A Cancer Journal for Clinicians, vol. 68, no. 6, pp. 394-424, 2018.

[26] D. Dellaportas, J. A. Gossage, and A. R. Davies, "Management and reconstruction of a gastroesophageal junction adenocarcinoma patient three years after pancreaticoduodenectomy: a surgical puzzle," Case Reports in Surgery, vol. 2016, Article ID 5650382, 3 pages, 2016.

[27] Y. Cheng, J. Zhang, L. Zhang, J. Wu, and Z. Zhan, "Enteral immunonutrition versus enteral nutrition for gastric cancer patients undergoing a total gastrectomy: a systematic review and meta-analysis," BMC Gastroenterology, vol. 18, no. 1, p. 11, 2018.

[28] Z. Khorgami, J. A. Petrosky, A. Andalib, A. Aminian, P. R. Schauer, and S. A. Brethauer, "Fast track bariatric surgery: safety of discharge on the first postoperative day after bariatric surgery," Surgery for Obesity and Related Diseases, vol. 13, no. 2, pp. 273-280, 2017.

[29] M. B. Esteban, P. Vorwald, S. Ortega Lucea, and J. M. Ramírez Rodríguez, "Rehabilitación multimodal en la cirugía de resección gástrica,” Cirugía Española, vol. 95, no. 2, pp. 73-82, 2017.

[30] R. Barlow, P. Price, T. D. Reid et al., "Prospective multicentre randomised controlled trial of early enteral nutrition for patients undergoing major upper gastrointestinal surgical resection," Clinical Nutrition, vol. 30, no. 5, pp. 560-566, 2011.

[31] F. Xin, S. A. S. Mzee, G. Botwe et al., "Short-term evaluation of immune levels and nutritional values of EN versus PN in gastric cancer: a systematic review and a meta-analysis," World Journal of Surgical Oncology, vol. 17, no. 1, p. 114, 2019.

[32] Q. S. Yu, Y. Y. Yuan, J. D. Liu et al., "[Effect of qihuang decoction on the intestinal mucosal immunologic barrier of rats after gastric resection]," Zhongguo Zhong Xi Yi Jie He Za Zhi, vol. 36, pp. 1358-1363, 2016.

[33] D. W. Chen, Z. W. Fei, Y. C. Zhang, J. M. Ou, and J. Xu, "Role of enteral immunonutrition in patients with gastric carcinoma undergoing major surgery," Asian Journal of Surgery, vol. 28, no. 2, pp. 121-124, 2005.

[34] Y. Liu and Yu Qing-sheng, "Effect of early use of medicinal herbs through intestinal canal on gastrointestinal peristalsis after gastric cancer operation," Zhong Guo Zhong Xi Yi Jie He Za Zhi, vol. 14, pp. 538-540, 2008.

[35] Q. S. Yu and H. L. Yu, "[Effect of qihuang decoction on mRNA expressions of Bcl-2, Bax, and Caspase-3, 9 in intestinal mucosa epithelium of ischemia/reperfusion injured rats]," Zhongguo Zhong Xi Yi Jie He Za Zhi, vol. 31, pp. 223-227, 2011.

[36] I. Abdikarim, X. Y. Cao, S. Z. Li et al., "Enhanced recovery after surgery with laparoscopic radical gastrectomy for stomach carcinomas," World Journal of Gastroenterology, vol. 21, no. 47, pp. 13339-13344, 2015.

[37] T. Inoue, R. Shinnakasu, W. Ise, C. Kawai, T. Egawa, and T. Kurosaki, "The transcription factor Foxol controls germinal center B cell proliferation in response to T cell help," The Journal of Experimental Medicine, vol. 214, no. 4, pp. 1181-1198, 2017.

[38] Q. S. Yu, Z. Chen, and X. Tang, "Effect of qihuang decoction on lymphocytic immune function after radical gastrectomy," Chinese Journal of Experimental Traditional Medical Formulae, vol. 2, pp. 13-15, 1996.

[39] C. S. Wang, Q. S. Yu, J. F. Pan, and etal, "Effect of qihuang decoction on proinflammatory cytokine and its mRNA expression in postoperative patients with upper gastrointestinal tract tumors," Zhongguo Zhong Xi Yi Jie He Wa Ke Za Zhi, vol. 18, no. 1, pp. 3-6, 2012.

[40] J. Yu, H. Ji, X. Dong, Y. Feng, and A. Liu, "Apoptosis of human gastric carcinoma MGC-803 cells induced by a novel Astragalus membranaceus polysaccharide via intrinsic mitochondrial pathways," International Journal of Biological Macromolecules, vol. 126, pp. 811-819, 2019. 
[41] W. Xu, R. Guan, F. Shi, A. Du, and S. Hu, "Structural analysis and immunomodulatory effect of polysaccharide from Atractylodis macrocephalae Koidz. on bovine lymphocytes," Carbohydrate Polymers, vol. 174, pp. 1213-1223, 2017.

[42] J. Jiao, J. Wu, M. Wang, C. Zhou, R. Zhong, and Z. Tan, "Rhubarb supplementation promotes intestinal mucosal innate immune homeostasis through modulating intestinal epithelial microbiota in goat kids," Journal of Agricultural and Food Chemistry, vol. 66, no. 4, pp. 1047-1057, 2018. 